In my experience also I believe that a history of thyroid enlargement and rheumatoid arthritis is extraordinarily common. When rou examine the patient there may be no evidence, but on inquiry into the past history it is astonishing how often you will get a history of goitre. I have recently had under treatment a case with the following family history.

Maternal grandmother suffered from goitre; mother had a goitre as a girl and died at 76 , bedridden with arthritis, high blood pressure, and attacks of angio-neurotic oedema. The patient has three sisters-all of whom suffered from Graves's disease, one developing the disease a week after marriage. A brother was laid up for a long time, and it was thought he was going to develop to 13 years of age he is now quite fit. The patient wot start e menstruating until she was 17 , when she had considerable dysmenorrhoea. At 19 the heart was said to be weak, and for the
last few years she has suffered from tachycardia, auricular flutter, last few years
and fibrositis.

Association of Pregnancy with Rheumatoid Arthritis.

For years $I$ have been struck with the fact that a patient with rheumatoid arthritis will give you the following history: that she became pregnant, and that during pregnancy she was very much better, but that during the puerperium all the symptoms returned with increased severity. This clinical fact has given me much thought. Now the thyroid is an active accelerator of metabolism, and its close connexion with reproduction is shown by its frequent physiological enlargement in the female-at puberty, marriage, and pregnancy, and sometimes at menstruation, and by its partial involution at the climacteric. During pregnancy great strain is thrown on the thyroid and the patient often becomes hypothyroidic for the time being. Then there is the association of the secretion of the corpus luteum and the pituitary, the internal secretion of the ovary stimulating the secretion of pituitrin; when the corpus luteum develops in pregnancy it checks this process in the interest of the foetus. Langdon Brown points out that the blood sugar curves of pituitary glycosuria and the glycosuria of pregnancy are similar, and suggests that the latter condition is due to pituitary disturbance, which is comprehensible, since pituitrin directly inhibits the action of insulin. This suggests that the normal holding up of pituitrin during pregnancy allows sugar to be stored up more readily in the interest of the offspring through the unchecked action of the insulin. Again, Cumberbatch and Robinson put forth the hypothesis that arthritis is directly or indirectly due to inactivity of the corpora lutea.

It is worth considering, therefore, whether the exhausted thyroid tends to reproduce the symptoms of rheumatoid disease, which have been held up in some way by the corpus luteum's inhibitory action on the posterior pituitary, and has thus restored the endocrine balance during pregnancy with amelioration of all the arthritic symptoms.

\section{Arthritis of the Menopause.}

The loss of the internal secretion of the ovary along with the partial involution of the thyroid produces a mild myxoedematous or hypothyroidic state. Clinically we know definitely that these cases improve with small doses of thyroid extract, some with the addition of ovarian extract and others with the addition of pituitary extract. I have particularly noticed how frequently these arthritic symptoms occur in cases where an artificial menopause has been induced and how improvement takes place under the above treatment.

Llewellyn ${ }^{12}$ holds that -there is a definite rheumatic diathesis due to endocrine instability, an instability which is observed in children and which is the forerunner of acute rheumatism. He bases his opinion on the following points, believing that:

1. The tendency of rheumatism to change its facies with the onset of puberty, the predilection of rheumatoid arthritis for the reproductive period and of osteo-arthritis and gout for the menopause or pre-senile epoch, suggest that the endocrine changes associated therewith account for the superficial diversity, but fundamental unity, of these affections.

2. Rheumatic children are all hypothyroidic.

3. Thyroid swelling very often ensues at the outset of acute rheumatism.

4. There is a definite geographical overlapping of goitre and acute rheumatism.
5. Acute rheumatism is an example of endocrine heredity. He quotes McCarrison : that the victims of simple toxic goitre are not only liable to Graves's disease, but, worse still, hand on to their children a condition of thyroid instability.

6. Both hypo- and hyper-thyroidism may follow acute rheumatism.

7. There is a higher incidence of rheumatism in goitrous than in non-goitrous children.

8. It is a clinical fact that some cases of acute rheumatism do not improve on salicylates until thyroid extract is added.

9. Subthyroidism spells suboxidation, and vasomotor instability implies irregularity in the supply and distribution of oxygen to the tissues.

Now, some may ask, where do infection and septic foci come in, and how is it that if you remove the septic foci the patient so frequently improves? The thyroid gland is one of the great defences against infection, and if the patient is already suffering from instability of the thyroid - cither hypo- or hyper-thyroidism-the presence of an infective focus will not improve matters, and its removal may, and probably does, in many cases restore stability, in the same way as the removal of septic tonsils is so often. the commencement of improvement in a case of Graves's disease.

Others may ask why it is that vaccines and protein shock are beneficial in these cases. Crammer answers this question when he says that the fact that many bacterial toxins stimulate the thyro-adrenal apparatus illustrates the curious and as yet unexplained phenomenon that in some infections the injection of non-specific vaccine, and even proteins, may have a striking therapeutic effect. The effect of the injection of these substances is to produce a typical rigor. The rationale of this so-called non-specific vaccint therapy, and protein therapy, would be that it elicits an increased functional activity from the thyroid adrenal apparatus-in fact, "sympathetic fever," which is one of the normal reactions of the organism against bacterial infection.

There is another point worthy of consideration-patients frequently tell us that their symptoms of rheumatoid arthritis commenced after a nervous shock, as an air raid, the death of a parent, an accident, etc. I have always believed that there is a psychological element in many cases of arthritis and endocrine imbalance, especially of the katabolic glands; their close association with the gonads supports this view. I have never been satisfied that septic foci were the sole causes of these diseases, but that behind it all there was a soil or diathesis which I believe to be connected with endocrine instability or imbalance.

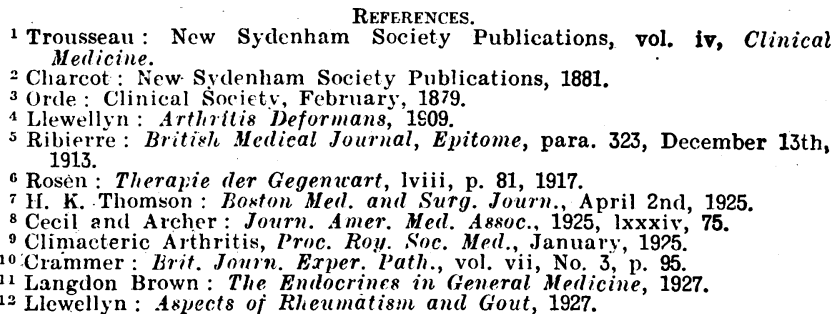

\section{SEA-SICKNESS AND ITS TREATMENT.} BY

\section{R. ALLAN BENNETT, M.D.Lond., M.R.C.P.,} SURGEON, PACIFIC STEAM NAVIGATION COMPANY.

SEA-sICKNESS in its milder forms may be regarded as an occasion for jesting by those whom it does not trouble, but for some sufferers its anticipation may poison the prospect of a voyage, and it may cause prolonged discomfort or even death. A woman, aged 40, rather debilitated by fever, travelling in a ship in which $I$ was a passenger many years ago, died after ten days of continual vomiting which no treatment could check. Such a catastrophe may be rare, but cases occur too frequently where suffering is prolonged for many days and the patient is reduced to a stato of extreme mental and physical prostration.

No age, no condition of life, is exempt. Old people going on a voyage for tho first time, sailors who have used the 
sea all their lives, young people and infants-all are liable, although children below the age of 12 seldom suffer severely. The type of vessel seems to have no bearing on the matter. One person is unaffected in a sailing boat and suffers in a liner; a sailor immune in a battleship may be overcome by the movement of a destroyer.

Undoubtedly the chief predisposing cause of sea-sickness is anticipation; quite a strong-minded person may dread a voyage, while an impressionable passenger, who has made up his mind that he is going to be sick, may be seen to turi pale even as he crosses the gangway. I doubt whether migraine predisposes to sea-sickness. If the determining cause was a labyrinthine vertigo, it would be reasonable to expect that any latent condition, with vertigo as a symptom, would increase such liability; but in my series I can trace no connexion between the two. Conversely, in diseases of the internal ear a relative immunity to accidental disturbances might be expected, and, indeed, persons with perforated drums, previously martyrs to sea-sickness, have been freed by their disability. On the other hand, I have known two cases of double mastoid disease associated with severe mal de mer.

The sole cause of sea-sickness is not a disturbance of the harmony that normally exists between the cerebellum, the labyrinth, the eye muscles, and elsewhere, and their co-ordination in the centres. Sea-sickness may be caused, before the ship leaves the dock, by some revalting sensory impression, such as the smell of the bilge in old days, and, in modern times, the characteristic mixture of rubber and engine oil that, pervades all but the largest vessels.

In a large proportion of cases there is chronic indigestion, with very often constipation, aggravated, maybe, by the excitement of preparing for the voyage, and, in cases where sea-sickness comes on late, by the excessive food consumed during the first days, which the stomach and bowels, weakened by lack of exercise, cannot deal with. In 88 out of 100 consecutive cases of sea-sickness the patients had over-eaten, either before or during the royage; 74 were constipated, while in 80 the condition was mild and vielded to twenty-four hours' starvation and calomel. In this milder type the attack begins as a rule with yawning or sighing; sooner or later there is nausea; and the last straw is supplied by the smell of a cigar or the careless remark of a bystander. Retching continues with an empty stomach; the hands and feet are icy; the face is pale, and a cold sweat breaks out upon the head and cheeks. The pulse is feeble and slow, and the blood pressure is sensibly lowered; there is headache, generally occipital, and dizziness, varying from a slight unsteadiness to a vertigo which makes it difficult for the patient to keep his feet. The tongue is furred, and the mouth is dry, with a bitter taste. If in these circumstances the patient can keep about in the air he can hold out, but in 12 of the series the vertigo was so distressing as to make this impossible.

The type of vertigo varies for no apparent reason; the patient may fancy that he is moving and the surrounding objects are stationary, or vice versa. Closing the eyes may improve things or make them worse; plugging the ears with cotton-wool may, or may not, help. In some patients the mental effect is slight; in other's there is a strong conviction that death is imminent, followed, as time goes on, by an unreasonable annoyance at its delay. In most cases, either as the result or in spite of the remedies given, the condition improves, and twenty-four or thirty-six hours will see the patient out of trouble. In a minority the condition may become serious: the romiting and retching continue for many days; hiccup may supervene, the rertigo become intense, the mental depression increase, and the patient be reduced to a state of weakness which may end in death.

As in sickness following excessive smoking, a disordered action of the pneumogastric nerve appears to be responsible. Our sense of position in space is a function of the vestibular nerve and its centres in the brain and cerebollum; the central connexions of its nucleus with those of the tenth and oculo-motor nerves is very close, and it may well be that all are implicated in the production of these symptoms.
But the earlier symptoms may fairly be attributed to the pneumogastric nerve. Ther may never pass beyond this stage; rertigo is not an invariable, and never an early, symptom of sea-sickness.

\section{Treaturent}

Much can be done in the way of prophylaxis. The traveller who starts his journey with the intention of being sick is seldom disappointed, and he who spends his last days ashore in feasting and farewells will reap what he has sown. If the one would moderate his appetite, and the other practise auto-suggestion for a week before sailing, there would be fewer tragedies during the first days of a voyage. Before a long journey by sea it is wise to take two meals a day-breakfast and dinner-for a period of seven days, eating meat at one onty, and avoiding all soups and madeup dishes. At midday take only bread and cheese and an apple. Take half the usual quantity of alcohol, drink water freely, and smoke but a little. The third day before sailing fast-eating nothing; but drinking water freely. That night and the following take a 5-grain blue pill, and the following mornings a Seidlitz powder or a dose of salts. No drugs are needed, but if the patient is exigent he may have a mixture three times a day containing 10 grains of bromide. Faith, however, is essential, and in the strength of it any man may go a-trawling forty days in the North Sea without discomfort. The trouble is that most people will make no effort to aroid a future inconvenience; they demand a cure without any help on their side.

During an attack of sea-sickness one remedy is as good as another if taken with confidence. Each traveller has lis own specific: one takes bromide or chloretone, or preparations of them under picturesque names; another clings to a peculiar form of belt; a third hangs an amulet about his neck; and a fourth plugs his ears with cotton-wool. In mild cases passenger's should be encouraged to persevere with their own " cures," exception being taken to nitroglycerin and amyl nitrite, which may lower dangerously the already diminished blood pressure. Failing such a private supply, 1 prescribe tincture of iodine, $1 \cdot \operatorname{minim}$ in a teaspoonful of milk every half-hour, and a mustard leaf to the pit of the stomach; biseuits and apples to eat; dry ginger ale to drink. However cruel it may seem, patients should be driven out of their cabins into the fresh air; only in the rarest cases is it necessary for them to stay in bed.

Such measures, with the help of time, will cure the majority of sufferers from sea-sickness, though the remainder may call for the most careful watching and tax every resource the ship provides. These others must be kepit in bed in an airy cabin, protected as far as possible from the smells and noises which cannot be avoided even in the best ships. Swinging cots are unsatisfactory; they must share to some extent the movements of the ship, and there is, in addition, a certain amount of joggling, caused by the "give" of the elastic fittings to the rods, which adds to the patient's discomfort. Hot bottles to the feet and a shade over the eves are comfortable to most people, and there "is virtue in "mustard leaves over the stomach and on the back of the neck.

Injections of adrenaline-10 minims, repeated if need be --are helpful in cases where the blood pressure falls below 105, and the same is true of stryehnine and camphor. Where romiting is incessant the ordinary gastric sedatives given by the mouth are useless: the patient should be encouraged to drink freely of hot water, with or without sodium bicarbonate, which will relieve the bitter taste so often experienced, and serve to wash away the mucus which is abundantly secreted. It may be necessary to give rertal feets, and nutrient suppositories are satisfying and convenient. 1 vithhold morphine as a hypnotic except as a last resource, for it is apt to cause increased nausea as the sedative effect passes away.

Finally, in cases where the exhaustion is becoming dangerous 1 have recourse to a method which $I$ have not seen mentioned. The patient is kept in a warm salt-water bath-temperature $90^{\circ}$ to $95^{\circ} \mathrm{F}$.- - for half an hour, an hour, or longer. The relief is great and remarkably prompt; it cannot be ascribed to the general sedative effect, 
but to the mechanical changing of the patient's environment. The specific gravity of the water is 1020 , and the body is supported very lightly on the buttocks, the shoulders, and the back of the head, with the toes just touching the end of the bath to prevent the legs from floating free. The bath itself shares the rolling and pitching of the ship, but the water it contains has not time to respond, and keeps its level, with the patient immersed in it partaking of its relative immobility. Watching a bathful of water in a rolling ship the surface appears to slant this way and that with the movement of the vessel, but a spirit: level floated on a cork raft will show that the water is unaffected. If the ship is pitching the long axis of the bath should be fore and aft; if rolling, athwart ship. The patient must not be able to see the apparent oscillation of the water, and the eyes should be bandaged-voluntary shutting is not enough, since in many cases vertigo is increased by the effort of closing the eyelids. If this procedure is carefully followed it always, in my experience, lessens the dizziness and gives relief, which is valuable in severe cases, and of ten permanent.

\section{FATAL SUPPRESSION OT URINE CAUSED BY LATENT HAEMAGGLUTININS.}

BY

GEOFFREY SHERA, M.A., M.D., B.Ch.CANTAB., M.R.C.S.,' L.R.C.P.;

pathologist to the pRincess alice MEMORIAL hospital, eastbotrNe, AND TO THE EAST SUSSEX CÖUNTY MENTAL HOSPITAL.

Following blood transfusion with an apparently compatible donor, the occurrence of a condition akin to blackwatei fever, both in its symptoms and morfid anatomy, and sometimes with a fatal issue, is fortunately uncommon. Blaker and Dodds $(1925)^{1}$ record with illuminating detail two such fatal cases, and add "rery valuable experimental observations. Keynes refers' to a case reported by Bernheim $^{3}$. where, after transfusion, haemoglobinuria oscurred, and progressively increased until the function of the kidney became so much interfered: with by dejósits of haemoglobin or damaged coipuseles that the patient died with suppression of urine and all the signs of uraemia? Dyke ${ }^{4}$ mentions two cases of haemoglobinuiria due to a Group 2 case being mistaken for a Group 4 owing to a low titre, Group 3 serum being -used for the prefiminary testing. Keynes ${ }^{2}$ also refers to non-fatal transient haemoglobinuria, and I myself have noted this phenomenon on two occasions.

It is of interest in this connexion to note that Yorke and Nauss, ${ }^{5}$ working on the pathology-of blackwater fever, injected rabbits with haemoglobin and produced intrarenal obstruction. Blaker and Dodds, repeating these experiments, found that in order to produce renal precipitation the urine must be acid and contain sodium chloride to the strength of at least 1 per cent.

Comparing three fatal cases, one of mine and.two of Blaker and Dodds, ${ }^{1}$ all have certain clinical features in common: onset. within a few hours; pyrexia $99^{\circ}$ to $104^{\circ}$; jaundice; methaemoglobinuria; and lumbar pain with symptoms of uraemia. One case was fatal in four days, my case in eight days, and the third in nineteen days. Nitrogen retention was apparent, and the cases took on the picture of latent uraemia.

One of the Blaker and Dodds cases showed a blood urea of $102 \mathrm{mg}$. per cent. on the fourth day, $178 \mathrm{mg}$. on the tenth day, $400 \mathrm{mg}$. on the sixteenth day, $618 \mathrm{mg}$. on the seventeenth day, and $803 \mathrm{mg}$. per cent. aftcr death. The second showed a blood urea of $287 \mathrm{mg}$. per cent.; and my case on the third day showed $174.6 \mathrm{mg}$. per cent.

\section{Clinical History of the Present Case.}

A woman, aged 36 , was admitted to the Princess Alice Memorial Hospital, under Mr. A. H. Crook, with a history, of five and a half weeks' amenorrhoea, except for a slight "loss" four days before admission. She had a black dischárge, with abdominal pain, constipation, and vomiting. She was very - blanched and restless; and had the obvious appearance of severe haemorrhage : of some duration. She was very tender and rigid in the left iliac fossa, and there was dullness in the flanks, with a fluid thrill. H Her

During the operation a pint of the husband's blood was transfused after preliminary cross-testing of the patient's serum against. the donor's cells for thirty minutes at room temperature and with the aid of the microscope: Both donor and patient werc: with the aid of the microscope: Both donor and patient werc
identified as Group 4 (Moss). At the operation the abdomen was identified as Group 4 (Moss). At the operation the abdomen was
opened by a median incision and much dark blood and clots oozed opened by a median incision and much dark blood and clots oozed
out, also a small foetus. The right Fallopian tube was found to be ruptured; it. was tied and removed. The clots and blood were swabbed out and the abdomen was closed. The wound remained clean subsequently, and the patient showed very slight signs of reaction, the temperature rising only to $100.8^{\circ}$.

\section{Aftcr-History}

On the next day (June 20th, 1927) the general condition of the patient was very good; she talked cheerfully, but slight icterus was noted, and there was complete suppression of urine. She complained of some abdominal pain, which was probably renal. A catheter was passed and 6 oz. of : blood-stained urine were obtained, full of blood debris. The pulse was 116, the respirations 24 , and the maximum temperature $99.5^{\circ}$

June 21st. : Suppression was complete. A catheter yielded cnly 2 oz. of blood-stained urine. The maximum temperature was $99.5^{\circ}$, the pulse 100 , and the respirations 24 .

June 22nd. Only $1 \frac{1}{2}$ oz. of urine were obtained by catheter. Hot-air baths, rectal salines, pilocarpine, and alkalis were given without 'effect. The blood urea was found to be $174.6 \mathrm{mg}$. per cent. The : pulse was 100 , the respirations 24 , the temperature cent. The pulse was 100, the respirations 24, the tempera
$99.2^{\circ}$, and the van den Bergh reaction was an indirect positive.

June 23 rd. Only $2 \frac{1}{2}$ oz. of urine were removed by catheter. Jumbar pain was now marked. The pulse was 92, the respirytions 22 ; and the temperature $98.6^{\circ}$.

Junc 24th... Only 1: oz. of urine oblained. Abdominal distension was noted, and diuretin was given without effect. The pulse was $88^{\circ}$, the respirations 20 , and the temperatur $984^{\circ}$.

June 25th. Signs of uraemia appeared in addition to haemolytic aundice; and the patient became drowsy and irritable: The systolic blood - pressure was 119 and the diastolic 78. The temperature was subnormal.

The patient died suddenly on June 27th.

\section{Post-mortem Examination.}

The necropsy was performed in my absence by the housesurgeon,-.Dr. J. F. Lang. Early adhesions were evident between the wound and the site of removal of the ruptured ectopic gesta tion. The kidneys were enlarged and of a pale coffee colour, with caporgement showing as striations in the medullary rays. The it contained some readily. The cortex was diminished in width it contained some engorged vessels as striated lines, but the mos striking feature was the marked engorgement of the medullary
rays. Microscopical sections revealed intense engorgement of the rays: Microscopical sections revealed intense engorgement of the
capilaries and tubules. Bowman's capsules gave evidence of comcapillaries and tubules. Bowman's capsules gave evidence of com-
pression, . and the tubular cells showed flattening and pressure atrophy with disappearance of many of the nuclei. The tubules were full of red blood cells or debris, and there was cloudy swelling where the condition was furthest advanced. The development: of fine, intertubular connecting tissue was visible. The kidney wass clearly the seat of advanced parenchymatous changes due to lack of nutrition of the cells, which was in turn due to piressure atrophy and toxic changes. Eosin-staining material in pressure atrophy and toxic changes. Eosin-staining material in masses and granules was evident in parts, The bladder contained
coffee-ground material. In regard to the liver, the bile ducts were coffee-ground material. In regard to the liver, the bile ducts were
markedly outlined macroscopically, and the picture of chronic venous congestion was present (nutmeg type). Microscopically there were similar: changes due to intense venous congestion with consequent pressure atrophy of the cells leading to parenchymatous degeneration, as evidenced by cell vacuolation and loss of nuclear staining. The spleen was very congested. Other organs were macroscopicaliy normal.

The cause of death was therefore presumably uraemia due to mechanical blockage of the kidneys and liver by blood cell agglutination proceeding slowly and progressively owing to the presence of small but effective amounts of agglutinogen in the patient's serum. Undetected incompatibility was obviously the cause. The patient and donor were apparently both Group 4, but a subsequent repetition of the cross-test (donor's cells and patient's serum) revealed at the end of sixty-five minutes a definite macroscopic agglutination. It will be remembered that thirty minutes had been allowed, and in the desperate condition of the case no longer delay was permissible. In the paper by Blaker and Dodds ${ }^{1}$ it. is recorded, in connexion with their first fatal case, that there had been some question as to the group interpretation, and, in regard- to the second, both donor and recipient were apparently Group 4, but subsequently a cross-test showed the patient's serum to agglntinate the donor's cells. On more than one occasion I have found that an apparently Group 4 donor's cells were agglutinated by a patient's serum (apparently also Group 4). A similar effect was recently noted by Dr. Dyson of St. Mary's Hospital. 